\title{
Accuracy of the Faddeev random phase approximation for light atoms
}

\author{
C. Barbieri* \\ Department of Physics, Faculty of Engineering and Physical Sciences, University of Surrey, Guildford, Surrey GU2 7XH, United Kingdom \\ and Theoretical Nuclear Physics Laboratory, RIKEN Nishina Center, 2-1 Hirosawa, Wako, Saitama 351-0198, Japan \\ D. Van Neck and M. Degroote \\ Center for Molecular Modeling, Ghent University, Technologiepark 903, B-9052 Gent, Belgium
}

(Received 10 May 2011; published 3 January 2012)

\begin{abstract}
The accuracy of the Faddeev random phase approximation (FRPA) method is tested by evaluating total and ionization energies in the basis-set limit. A set of light atoms up to Ar is considered. Comparisons are made with the results of coupled-cluster singles and doubles (CCSD), with third-order algebraic diagrammatic construction [ADC(3)], and with the experiment. It is seen that even for two-electron systems, $\mathrm{He}$ and $\mathrm{Be}^{2+}$, the inclusion of RPA effects leads to satisfactory results, and therefore it does not overcorrelate the ground state. The FRPA becomes progressively better for larger atomic numbers, where it gives $\approx 5 \mathrm{mH}$ more correlation energy, and it shifts ionization potentials by $2-10 \mathrm{mH}$ with respect to the similar ADC(3) method. The ionization potentials from FRPA tend to reduce the discrepancies with the experiment.
\end{abstract}

DOI: 10.1103/PhysRevA.85.012501

PACS number(s): $31.10 .+\mathrm{z}$

\section{INTRODUCTION}

$A b$ initio studies of electronic systems aim at a direct solution of the Schrödinger equation in terms of the underlying Coulomb interaction, thus avoiding phenomenological input [1]. Due to its favorable accuracy and the gentle scaling of computational requirements with increasing number of particles the single-reference coupled-cluster (CC) method [2] has become the most frequently used theory in contemporary investigations of molecular and atomic systems. One can thus calculate molecules for which full configuration interaction (FCI) would not be feasible. Another approach with analogous characteristics is the Green's function (GF) theory (or, equivalently, propagator theory) [3-6]. An early scheme based on this approach is the outer-valence GF (OVGF) [7,8], which expands ionization energies up to third order in perturbation theory. The OVGF is very practical and computationally simple, and it has found several applications to studies of ionization spectra [8-12]. However, it becomes inaccurate whenever inner- and outer-valence ionization energies (IEs) are subject to shake-up contaminations [13-15]. In such cases one needs to resort at least to the third-order algebraic diagrammatic construction [ADC(3)] method [16,17]. The $\operatorname{ADC}(n)$ approach is an intermediate-state representation $[18,19]$ of the GF made to be consistent with perturbation theory up to order $n$. Thus, it is size consistent and can be systematically improved by going to higher orders. For the one-body propagator, $\mathrm{ADC}(3)$ implies performing explicit configuration mixing between valence electrons and shake-up configurations, such as two-hole-one-particle ( $2 \mathrm{~h} 1 \mathrm{p})$ and/or two-particle-one-hole (2p1h) configurations [20]. These states are mixed together by $\mathrm{ADC}(3)$ theory in a TammDancoff approximation (TDA) fashion. The accuracy of the ADC(3) approach has been tested in several studies for both IEs [20,21] and excited states [22,23].

\footnotetext{
*Present address: University of Surrey, Guildford GU2 7XH, United Kingdom. Email: c.barbieri@ surrey.ac.uk
}

Green's function theory has also extensive applications to solid-state physics, where the most successful scheme is the GW approximation (GWA) [24-26]. The GWA describes the modification of electrons through repeated interactions with collective particle-hole (ph) excitations of the system, which are described in the random phase approximation (RPA). The RPA is essential for extended systems because it screens the Coulomb interaction at large distances [5,6] and it guarantees finite correlation energies in metals and the uniform electron gas [27-30]. In contrast, the TDA plasmon spectrum is incorrect and even diverges at small momenta. The GWA, however, is not always satisfactory. As an example, particle-particle (pp) and hole-hole (hh) configurations, which would be included by ADC(3) but not in GWA, are necessary to explain satellite structures above and below the Fermi surface [31]. How to include these effects efficiently in GWA is still being researched [32]. Conversely, the inclusion of RPA in atomic and molecular studies may be advantageous for describing long-range (van der Waals) forces and dissociation processes [33-35]. Thus, a practical method that combines ADC(3) with RPA might become beneficial to the fields above.

In a recent publication we have considered the $a b$ initio calculation of the Ne atom using Green's function theory in the so-called Faddeev random phase approximation (FRPA) [36], which was originally proposed for studies of nuclear structure [37-41]. This approach completely includes $2 \mathrm{p} 1 \mathrm{~h}$ and $2 \mathrm{~h} 1 \mathrm{p}$ states in the self-energy but expands these configurations in terms of couplings between valence particles and (simpler) $\mathrm{ph}$ and $\mathrm{pp}(\mathrm{hh})$ excitations that are calculated using RPA. By calculating these excitations in TDA one would be led back to the ADC(3) scheme. Stated otherwise, the FRPA can be seen as an extension of ADC(3) that employs RPA for pairs of electron and hole excitations (see also Ref. [42] for a discussion). The inclusion of RPA opens a possible way for treating long-range correlations in both finite and extended electron systems on a equal footing. An application of this method to extended systems, such as the electron gas, could be pursued following the approach of Ref. [43]. Before this is attempted, however, one wishes to better assess the quality of the method for 
light systems. A first study carried out on simple molecules has found accurate results and confirmed that potential RPA instabilities do not arise at equilibrium internuclear distances [44]. However, calculations were limited to small bases, and a proper investigation of the complete basis-set limit is still in order. This work addresses this last question by applying FRPA to a set of light atoms and using correlation-consistent bases for extrapolating to the basis-set limit.

The field of high-precision atomic structure calculations has a rich history. In particular multiconfiguration self-consistent field methods [45-50] have been shown to provide very accurate results. Using explicitly correlated Gaussian basis functions [51] is another technique to generate results of high quality. The method investigated in the present paper is not multireference in character, and as such, it is most closely related to single-reference $\mathrm{CC}$ methods; hence all our results will be compared to calculations at this level of theory.

There have been efforts to understand the connection between Green's function theory and CC theories. A study on the relationship between the ADC method and $\mathrm{CC}$ linear response showed how one can get from the approximate $\mathrm{CC}$ doubles (CC2) to the $\mathrm{ADC}(2)$ stability matrix by replacing the $\mathrm{CC} 2$ ground-state amplitudes by the ones obtained in first-order perturbation theory and taking the Hermitian average [52]. The relation between direct RPA and ring CC doubles (ring CCD) [53] is another example. Even though the correspondence between $\mathrm{CC}$ on the one side and $\mathrm{ADC}(3)$ and FRPA on the other side is not a priori clear, one can argue that $\mathrm{CC}$ singles and doubles (CCSD) has the closest resemblance to ADC(3) and FRPA and will be our reference.

The essential features of FRPA are reviewed in Sec. II so that this paper is self-contained. References to the details of the formalism are also given. In our calculations we adopt Gaussian basis sets and discuss the accuracy of extrapolations to the basis-set limit in Sec. III A. The results for total energies and IEs are given in Sec. III B, and the major conclusions are summarized in Sec. IV.

\section{FORMALISM}

\section{A. General propagator theory}

In the present study we consider the calculation of the single-particle propagator $[4,6]$,

$$
g_{\alpha \beta}(\omega)=\sum_{n} \frac{\left(z_{\alpha}^{n}\right)^{*} z_{\beta}^{n}}{\omega-\varepsilon_{n}^{+}+i \eta}+\sum_{k} \frac{z_{\alpha}^{k}\left(z_{\beta}^{k}\right)^{*}}{\omega-\varepsilon_{k}^{-}-i \eta},
$$

where $\alpha, \beta, \ldots$, label a complete orthonormal basis and

$$
\begin{aligned}
& z_{\alpha}^{n}=\left\langle\Psi_{n}^{N+1}\left|c_{\alpha}^{\dagger}\right| \Psi_{0}^{N}\right\rangle, \quad \varepsilon_{n}^{+}=E_{n}^{N+1}-E_{0}^{N}, \\
& z_{\alpha}^{k}=\left\langle\Psi_{k}^{N-1}\left|c_{\alpha}\right| \Psi_{0}^{N}\right\rangle, \quad \varepsilon_{k}^{-}=E_{0}^{N}-E_{k}^{N-1} .
\end{aligned}
$$

In these definitions, $c_{\alpha}\left(c_{\beta}^{\dagger}\right)$ are second-quantization annihilation (creation) operators, $\left|\Psi_{n}^{N+1}\right\rangle,\left|\Psi_{k}^{N-1}\right\rangle$ are the eigenstates, and $E_{n}^{N+1}, E_{k}^{N-1}$ are the eigenenergies of the $(N \pm 1)$-electron system. Therefore, the poles of the propagator reflect the electron affinities (EAs) and IEs. Equation (1) also yields the total binding energy via the Migdal-Galitskiı̌-Koltun sum rule [6],

$$
E_{0}^{N}=\frac{1}{2} \sum_{k} \sum_{\alpha, \beta}\left(u_{\alpha \beta}+\varepsilon_{k}^{-} \delta_{\alpha \beta}\right) z_{\beta}^{k}\left(z_{\alpha}^{k}\right)^{*},
$$

where $u_{\alpha \beta}$ represent the matrix elements of the one-body part of the Hamiltonian (kinetic energy plus nuclear attraction) and the $k$ sum runs only over the eigenstates of the $(N-1)$ electron system.

The one-body Green's function solves the Dyson equation (hereafter, summations over repeated indices are implied)

$$
g_{\alpha \beta}(\omega)=g_{\alpha \beta}^{0}(\omega)+g_{\alpha \gamma}^{0}(\omega) \Sigma_{\gamma \delta}^{\star}(\omega) g_{\delta \beta}(\omega),
$$

where $g^{0}(\omega)$ is the propagator for a free particle. The irreducible self-energy $\Sigma_{\gamma \delta}^{\star}(\omega)$ acts as an effective, energydependent, potential that can be written as $[54,55]$

$$
\begin{aligned}
\Sigma_{\alpha \beta}^{\star}(\omega)= & \Sigma_{\alpha \beta}^{\infty}+\tilde{\Sigma}_{\alpha \beta}(\omega)=\int \frac{d \omega}{2 \pi i} V_{\alpha \gamma, \beta \delta} g_{\delta \gamma}(\omega) e^{-i \omega \eta^{+}} \\
& +\frac{1}{4} V_{\alpha \lambda, \mu \nu}\left[R_{\mu \nu \lambda, \mu^{\prime} \nu^{\prime} \lambda^{\prime}}^{(2 \mathrm{p} 1 \mathrm{~h})}(\omega)+R_{\mu \nu \lambda, \mu^{\prime} \nu^{\prime} \lambda^{\prime}}^{(2 \mathrm{~h} 1 \mathrm{p})}(\omega)\right] V_{\mu^{\prime} \nu^{\prime}, \beta \lambda^{\prime}},
\end{aligned}
$$

and $V_{\alpha \beta, \gamma \delta}$ are antisymmetrized Coulomb matrix elements. In Eq. (5) we have emphasized the energy-independent [or correlated Hartree-Fock (CHF)] contribution to the self-energy $\Sigma^{\infty}$. This generalizes the Hartree-Fock (HF) potential by replacing the Slater mean field with the (correlated) density matrix extracted from the dressed propagator (1). $\Sigma^{\infty}$ is represented by the first diagram on the right-hand side in Figs. 1(a) and 1(b). The remaining term, $\tilde{\Sigma}(\omega)$, accounts for deviations from the mean field and depends on the $2 \mathrm{p} 1 \mathrm{~h}$ and $2 \mathrm{~h} 1 \mathrm{p}$ polarization propagators, $R^{(2 \mathrm{p} 1 \mathrm{~h})}(\omega)$ and $R^{(2 \mathrm{~h} 1 \mathrm{p})}(\omega)$. These involve the simultaneous propagation of $2 \mathrm{p} 1 \mathrm{~h}$ (or $2 \mathrm{~h} 1 \mathrm{p}$ ) and higher excitations. Equation (5) is represented in Fig. 1(a) in terms of time-ordered Feynman diagrams (or Goldstone diagrams). $R(\omega)$ can also be expanded in terms of Coulomb

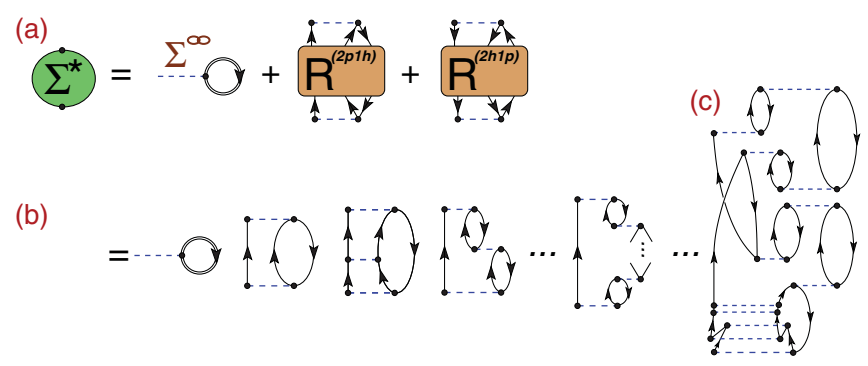

FIG. 1. (Color online) (a) The self-energy $\Sigma^{\star}(\omega)$ separates exactly into a correlated $\mathrm{HF}$ term $\Sigma^{\infty}$ and the polarization propagators $R^{(2 \mathrm{p} 1 \mathrm{~h} / 2 \mathrm{~h} 1 \mathrm{p})}(\omega)$ for the $2 \mathrm{p} 1 \mathrm{~h}$ and $2 \mathrm{~h} 1 \mathrm{p}$ motion. (b) Upon expansion of $R(\omega)$ in Feynman diagrams, one obtains this series of diagrams for the self-energy. (c) Another, more complicated, term appearing in the expansion of $R^{(2 \mathrm{p} 1 \mathrm{~h})}(\omega)$. This is also included in the FRPA contribution of Fig. 3, but it introduces a time ordering not generated by FTDA or ADC(3). Dashed lines are antisymmetrized Coulomb matrix elements $V_{\alpha \beta, \gamma \delta}$, single lines represent the reference state (a HF propagator), and the double lines represent the correlated propagator of Eq. (1). All diagrams are time ordered, with time propagating upward. 


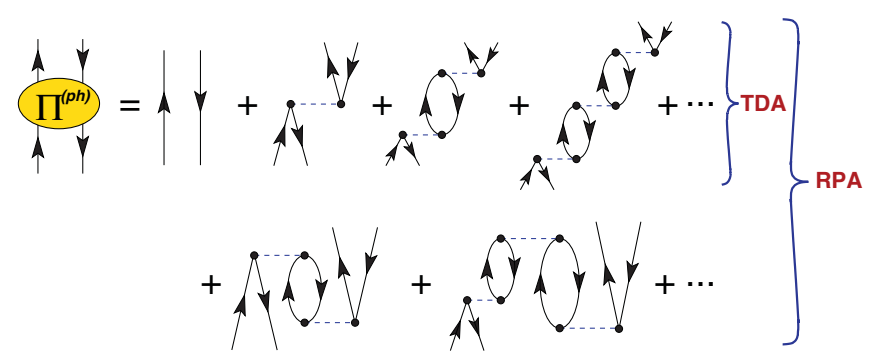

FIG. 2. (Color online) Expansion of the ph propagator $\Pi(\omega)$ in a series of ring diagrams. The diagrams resummed by TDA are shown in the first line. The second line gives examples of time-inversion patterns that are generated only by RPA; these account for the presence of $2 \mathrm{p} 2 \mathrm{~h}$ and more complicated configurations in the correlated ground state. The diagrams are time ordered, with time propagating upward.

matrix elements and unperturbed propagators, as shown in Figs. 1(b) and 1(c).

\section{B. The Faddeev random-phase-approximation method}

The Faddeev approach consists in expanding $R^{(2 \mathrm{p} 1 \mathrm{~h})}$ and $R^{(2 \mathrm{~h} 1 \mathrm{p})}$ in terms of couplings between single electrons or holes and $\mathrm{ph}, \mathrm{pp}$, or hh excitations [36,56]. Information about the latter is fully contained in the ph polarization propagator $\Pi(\omega)$ that describes excited states of the $N$-electron system and the two-particle propagator $g^{I I}(\omega)$ that describes the addition or removal of two electrons. These Green's functions are calculated as resummations of ring and ladder diagrams using either the TDA or the RPA. The difference between the two approximations is in the choice of the propagators for the noninteracting ph (i.e., $\Pi^{0}$ ) and pp (hh) (i.e., $g^{0}$ ) configurations. Both of these contain a term that conserves the direction of time propagation and one that inverts it. By including only the first term the TDA equations are obtained. If both contributions are retained, one is led to the RPA. The RPA induces extra time orderings in the resummations, as shown in Fig. 2 for the ph case. These account for $2 \mathrm{p} 2 \mathrm{~h}$ and more complicated admixtures in the ground state that are generated by correlations.

The $R^{(2 \mathrm{p} 1 \mathrm{~h})}(\omega)$ and $R^{(2 \mathrm{~h} 1 \mathrm{p})}(\omega)$ propagators are obtained by first calculating $\Pi(\omega)$ and $g^{I I}(\omega)$ and then by recoupling them to single-electron or -hole states, as shown in Fig. 3. This is done by solving the set of Faddeev equations detailed in Refs. [36,56]. We refer to this procedure as Faddeev TDA (FTDA) or Faddeev RPA depending on the approximation chosen to calculate $\Pi(\omega)$ and $g^{I I}(\omega)$. The Faddeev summation and the diagram depicted in Fig. 3 are the same in both cases. Contributions from ph and pp (hh) excitations in all possible partial waves are included in FRPA and FTDA since this is required for a complete solution of the problem. In order to fulfill Pauli constraints up to $2 \mathrm{p} 1 \mathrm{~h}$ and $2 \mathrm{~h} 1 \mathrm{p}$ level in the expansion for $R(\omega)$ one must employ the generalized version of RPA (in which the Coulomb matrix elements are the antisymmetrized ones). Two separate sets of Faddeev equations are used for $R^{(2 \mathrm{p} 1 \mathrm{~h})}(\omega)$ and $R^{(2 \mathrm{~h} 1 \mathrm{p})}(\omega)$.

The Faddeev procedure can be recast in terms of two separate diagonalizations in the $2 \mathrm{p} 1 \mathrm{~h}$ and $2 \mathrm{~h} 1 \mathrm{p}$ configuration spaces. In each case one solves a non-Hermitian eigenvalue problem [56]. The Dyson equation is transformed into a
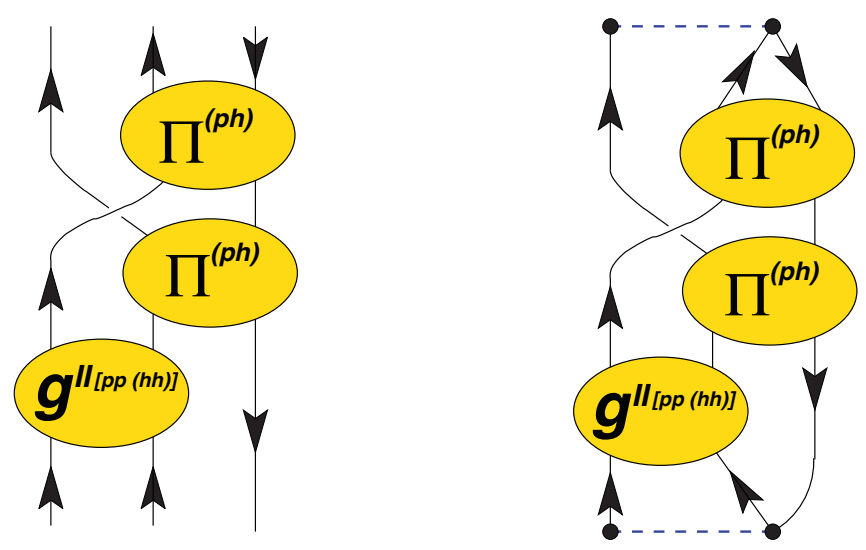

FIG. 3. (Color online) (left) Example of one of the diagrams for $R^{(2 \mathrm{plh})}(\omega)$ that are summed to all orders by means of the Faddeev method. Each of the ellipses represents an infinite sum of rings $[\Pi(\omega)]$ or ladders $\left[g^{I I}(\omega)\right]$. Contributions of all possible partial waves are included. (right) The corresponding contribution to the self-energy, obtained upon insertion of $R^{(2 \mathrm{plh})}(\omega)$ into Eq. (5). This representation is valid for both the FTDA and FRPA approaches, the difference between the two being in the diagrams implicitly resummed inside $\Pi(\omega)$ and $g^{I I}(\omega)$ (see Fig. 2).

diagonalization problem, which can be solved directly for the IE and EA and the corresponding residues in the single-particle propagator (1). The explicit form of the FTDA and FRPA equations is given in Ref. [36], and their reduction to a matrix diagonalization is described in Ref. [56]. In the particular case of the FTDA, one finds that the Faddeev Hamiltonian matrix is Hermitian and is equivalent to the effective interaction among $2 \mathrm{p} 1 \mathrm{~h}$ or $2 \mathrm{~h} 1 \mathrm{p}$ states in $\operatorname{ADC}(3)$. When the coupling vertex between single-particle states and $2 \mathrm{p} 1 \mathrm{~h}$ and $2 \mathrm{~h} 1 \mathrm{p}$ states, in analogy with $\mathrm{ADC}(3)$ theory, is taken as the minimum coupling that ensures consistency with perturbation theory up to third order [17], the equations reduce to the usual Dyson ADC(3) equations [44]. In the following we refer to this approximation as "FTDA" with the understanding that for the present calculations it is strictly equivalent to "ADC(3)".

\section{RESULTS}

We considered a set of neutral atoms and ions corresponding to shell and subshell closures with $Z \leqslant 18$. The calculations of the smallest systems ( $\mathrm{He}, \mathrm{Be}$, and $\mathrm{Be}^{2+}$ ) were performed using the correlation-consistent polarization valence Gaussian bases, cc-pVX $Z$, of increasing quality from double to quintuple zeta $(X=2-5)$. For the larger atoms it was found that a sizable fraction of the correlation energy is lost with similar bases. The remaining systems were therefore calculated with the corresponding core-valence bases, cc-pCVX $Z$, which include additional compact Gaussians to improve the description of the core electrons. This choice was seen to speed up the convergence and led to accurate results for these atoms. ${ }^{1}$ The correction to the correlation energies induced by the extra core orbits

\footnotetext{
${ }^{1}$ The augmented version of the bases (aug-cc-pVXZ) were also tested and gave no sizable improvement for the quantities being considered in this work.
} 
TABLE I. Total binding energies (in hartrees) for Ne and Be obtained for cc-p(C)VXZ bases of different sizes. The results obtained with FTDA and FRPA (with self-consistency in the CHF diagram) and with the CCSD methods are compared to FCI calculations.

\begin{tabular}{|c|c|c|c|c|c|}
\hline \multirow[b]{2}{*}{$E_{\text {tot }}$} & \multirow{2}{*}{$\begin{array}{c}\mathrm{Ne} \\
\text { cc-pCVDZ }\end{array}$} & \multicolumn{4}{|c|}{$\mathrm{Be}$} \\
\hline & & cc-pVDZ & cc-pVTZ & cc-pVQZ & cc-pV5Z \\
\hline FTDA & -128.7191 & -14.6089 & -14.6154 & -14.6314 & -14.6375 \\
\hline FRPA & -128.7210 & -14.6084 & -14.6150 & -14.6310 & -14.6371 \\
\hline CCSD & -128.7211 & -14.6174 & -14.6236 & -14.6396 & -14.6457 \\
\hline Full CI & -128.7225 & -14.6174 & -14.6238 & -14.6401 & -14.6463 \\
\hline
\end{tabular}

increases with the number of electrons, and it was found to be $\approx 40 \mathrm{mH}$ for $\mathrm{Ne}$ and $\approx 300 \mathrm{mH}$ for $\mathrm{Mg}$ in the cc-pCVQZ basis.

The bases for the $\mathrm{Be}^{2+}\left(\mathrm{Mg}^{2+}\right)$ ions were obtained from the cc-p $(\mathrm{C}) \mathrm{VXZ}$ sets for $\mathrm{He}(\mathrm{Ne})$ but scaling the corresponding single-particle orbits to correct for the different atomic number,

$$
\phi_{\mathrm{Be}^{2+} / \mathrm{Mg}^{2+}}^{i}(r) \propto \phi_{\mathrm{He} / \mathrm{Ne}}^{i}\left(r \frac{Z}{N}\right),
$$

where $Z$ is the nuclear charge and $N=Z-2$ is the number of electrons.

Correlation and ionization energies were computed with both the FTDA and the FRPA methods. The CHF term $\Sigma^{\infty}$ was calculated fully self-consistently by iterating Eq. (4) and the first term on the right-hand side in Eq. (5) [the first diagram in Figs. 1(a) and 1(b)]. ${ }^{2}$ This aspect is important since it consistently generates all the nondynamic contributions from perturbation theory up to third order and more. The dynamic part of the self-energy $\tilde{\Sigma}(\omega)$ was instead calculated in terms of an HF reference state.

The ground-state energies are compared to the results of CCSD, starting from the same HF reference state. In all cases, the full configuration space was considered to be active (i.e., no frozen-core approximation). The ground-state energies are compared to empirical values, which have been corrected by subtracting relativistic effects [57-59]. Ionization energies are compared to experimental spectra. Since our calculations are nonrelativistic, it is of interest to have an estimate of relativistic effects. This was studied at the level of the ADC(3) formalism in Ref. [60], and one expects the error at the FRPA level to be of the same order of magnitude. For instance, in $\mathrm{Ne}$ the $2 p$ ionization energy is shifted by the small amount of 0.8 millihartree, and the same holds for the $3 p$ state of $\mathrm{Ar}$ (a shift of 0.6 millihartree). The $s$ states are reported to show larger effects and are shifted upward by $5.1 \mathrm{mH}$ for the $2 s$ state of $\mathrm{Ne}$ and by $6.6 \mathrm{mH}$ for the $3 s$ state of Ar; i.e., in this case the effects become comparable to the discrepancy between experimental and theoretically obtained values.

\section{A. Convergence}

Total binding energies predicted by both Green's function theory and CCSD are compared with the results of full

\footnotetext{
${ }^{2}$ The present notation differs from previous works, where FRPA denoted the $\Sigma^{\infty, H F}$ obtained from the HF reference state and FRPAc denoted the fully correlated CHF version of it $[36,44]$. In this paper we drop the subscript $\mathrm{c}$ and always use FRPA to denote the complete CHF term.
}

configuration interaction (FCI) in Table I. For a $\mathrm{Ne}$ atom in cc-pCVDZ, Green's functions and CCSD agree with each other and deviate from the exact result by less than $2 \mathrm{mH}$. FRPA gives just a very small correction, but it halves the discrepancy between FTDA and FCI. The total correlation energy for this basis is $233 \mathrm{mH}$. The atom of $\mathrm{Be}$ is the most difficult case among those discussed here due to the fact that this is not a good closed-shell system. In this case, a near degeneracy between the $2 s$ and $2 p$ orbitals leads to very soft excitations of the $J^{\pi}=1^{-}, S=1$ states and drives the ph-RPA equations close to instability. The FRPA was thus solved by employing the TDA approximation of the polarization propagator in this channel alone (all other partial waves were treated properly in RPA). The resulting correlation energies agree with FTDA, indicating that RPA is not crucial for this small system nor does it introduce spuriousities by overcorrelating the ground state. FCI calculations of Be were possible for all bases up to quintuple zeta and are reproduced by CCSD with high accuracy. However, FTDA and FRPA are consistently behind by about $9 \mathrm{mH}$, corresponding to $10 \%$ of the total correlation energy. This is the most serious discrepancy obtained in this work and suggests a limitation of the FRPA, in its present form, for near-degenerate systems. To overcome this, it may be necessary to improve the effective coupling vertex (which is described with higher accuracy by CCSD) or to extend the treatment of the excitation spectrum of the polarization propagator beyond bare ph states [61]. ${ }^{3}$ The close agreement between FTDA and FRPA in Table I is a welcome feature since for a few electrons in the Be atom one should expect RPA-like effects (that is, several ph excitations admixed in the ground state) to be negligible. The RPA is not optimal for few-body systems since breaking of the Pauli principle may become substantial in these cases. Nevertheless, this result (and the one for He discussed below) suggests that it can be safely applied also in this regime without overestimating ground-state correlations. The usual issues of RPA for cases of near degeneracy remain and may lead to instabilities in certain partial waves, as described above. In the worst case it is still possible to release the RPA requirement in such channels and obtain reasonable results.

Extrapolations to the basis-set limit were obtained from two consecutive sets according to

$$
E_{X}=E_{\infty}+A X^{-3}
$$

\footnotetext{
${ }^{3}$ In the ADC language, this means adding fourth- and fifth-order terms that are introduced at the $\mathrm{ADC}(5)$ level.
} 
TABLE II. Convergence of total energies and IEs (in hartrees) in the FRPA approach. Calc. indicates energies calculated using double $(X=\mathrm{D})$ to quintuple $(X=5)$ valence orbits basis sets. Extrap. indicates results extrapolated from two consecutive sets using Eq. (7). The $\mathrm{Be}$ atom was calculated with the cc-pVXZ bases, while $\mathrm{Ne}, \mathrm{Mg}$, and Ar were done using cc-pCVXZ. The experimental values are from Refs. [57-59,62].

\begin{tabular}{|c|c|c|c|c|c|c|c|}
\hline & & & $\mathrm{cc}-\mathrm{p}(\mathrm{C}) \mathrm{VDZ}$ & cc-p(C)VTZ & cc-p(C)VQZ & cc-p(C)V5Z & Experiment \\
\hline \multirow[t]{6}{*}{$E_{\mathrm{tot}}$} & $\mathrm{Be}$ & calc. & -14.6084 & -14.6150 & -14.6310 & -14.6371 & -14.6674 \\
\hline & & extrap. & & -14.6178 & -14.6427 & -14.6436 & \\
\hline & $\mathrm{Ne}$ & calc. & -128.7210 & -128.8643 & -128.9079 & -128.9226 & -128.9383 \\
\hline & & extrap. & & -128.9246 & -128.9397 & -128.9381 & \\
\hline & $\mathrm{Mg}$ & calc. & -199.8147 & -199.9507 & -200.0033 & -200.0271 & -200.054 \\
\hline & & extrap. & & -200.0080 & -200.0417 & -200.0519 & \\
\hline \multirow[t]{4}{*}{ IE } & $\operatorname{Ar}(3 p)$ & calc. & 0.5623 & 0.5695 & 0.5751 & 0.5770 & 0.579 \\
\hline & & extrap. & & 0.5725 & 0.5792 & 0.5788 & \\
\hline & $\operatorname{Ar}(3 s)$ & calc. & 1.0985 & 1.0616 & 1.0599 & 1.0622 & 1.075 \\
\hline & & extrap. & & 1.0461 & 1.0586 & 1.0646 & \\
\hline
\end{tabular}

where $X$ is the cardinal number of the basis. This relation is known to give proper extrapolations for correlation energies [1]. Table II gives some examples of the calculated binding energies for all basis sizes and shows the convergence of the extrapolated results. In the smallest systems, up to Ne, we find changes of less than $2 \mathrm{mH}$ between the last two extrapolations ( $X=T, Q$ and $X=Q, 5$ ). This number can be taken as a measure of the uncertainty in reaching the basis-set limit. For the larger atoms $\mathrm{Mg}$ is the one that converges more slowly, with a difference of $10 \mathrm{mH}$ (we found $7 \mathrm{mH}$ for Ar). Calculations with $X=6$ are beyond our present computational capabilities. However, given the fast convergence with increasing cardinal number, it appears safe to assume an uncertainty of $\leqslant 5 \mathrm{mH}$ for $\mathrm{Mg}$ and $\mathrm{Ar}$.

In general, IEs and EAs tend to converge faster because they represent differences of total energies between the $N$-electron ground state and the excited states of $(N \pm 1)$ electrons. Inaccuracies in the correlation energies are similar and therefore could cancel each other to a large extent. Equation (7) is preserved when taking differences of correlation energies that obey the same trend, and therefore one may expect that a similar behavior applies to IEs for large basis sets. However, this is not always guaranteed, especially in cases where shake-up configurations are important. For smaller bases these contributions are less stable with respect to changing basis set and can affect IEs differently. The possible behaviors are displayed in Table II for the calculated IEs of Ar. The $3 p$ orbit has a strong one-hole character and converges smoothly. Here the difference of only $0.4 \mathrm{mH}$ between the last two extrapolations indicates that a convergence as $X^{-3}$ effectively takes place. We obtained similar trends for the other cases. The only remarkable exception is the $3 s$ hole in Ar, which has a large admixture of $2 \mathrm{~h} 1 \mathrm{p}$ configurations. The calculated IE shows an oscillatory behavior; however, a monotonic convergence could still happen for larger bases once shake-up contributions have stabilized. In Sec. III B, we will apply Eq. (7) also to extrapolate ionization energies. We estimate an error up to $2 \mathrm{mH}$ for the larger atoms and $<1 \mathrm{mH}$ for the smaller ones.

\section{B. Ground states and ionization energies of simple atoms}

Table III shows the ground-state energies extrapolated from $X=Q, 5$ for both Green's function and CCSD methods. These are compared to the corresponding Hartree-Fock results and the experiment. The empirical values are from Refs. [57-59] and have been corrected by subtracting relativistic effects. The CCSD results for $\mathrm{He}$ and $\mathrm{Be}^{2+}$ are equivalent to FCI. Note that the extrapolated value based on $X=Q$ and $X=5$ is not yet fully converged at the submillihartree level and lies slightly below the basis-set limit. We see that FRPA misses $1 \mathrm{mH}$, or $2 \%$, of the correlation energy of He. In larger systems

TABLE III. Hartree-Fock, FTDA, FRPA, and CCSD total energies (in hartrees) extrapolated from the cc-p(C)VQZ and cc-p(C)V5Z basis sets. $\mathrm{He}, \mathrm{Be}^{2+}$, and $\mathrm{Be}$ were calculated with the cc-pVXZ bases, while cc-pCVXZ bases were used for the remaining atoms. The deviations from the experiment are indicated in parentheses (in mhartrees). The experimental energies are from Refs. [57-59]. The rms errors in parentheses are calculated by neglecting the Be results.

\begin{tabular}{|c|c|c|c|c|c|}
\hline & Hartree-Fock & FTDA & FRPA & CCSD & Experiment \\
\hline $\mathrm{He}$ & $-2.8617(+42.0)$ & $-2.9028(+0.9)$ & $-2.9029(+0.8)$ & $-2.9039(-0.2)$ & -2.9037 \\
\hline $\mathrm{Be}^{2+}$ & $-13.6117(+43.9)$ & $-13.6559(-0.3)$ & $-13.6559(-0.3)$ & $-13.6561(-0.5)$ & -13.6556 \\
\hline $\mathrm{Be}$ & $-14.5731(+94.3)$ & $-14.6438(+23.6)$ & $-14.6436(+23.8)$ & $-14.6522(+15.2)$ & -14.6674 \\
\hline $\mathrm{Ne}$ & $-128.5505(+387.8)$ & $-128.9343(+4.0)$ & $-128.9381(+0.2)$ & $-128.9353(+3.0)$ & -128.9383 \\
\hline $\mathrm{Mg}^{2+}$ & $-198.837(+444)$ & $-199.226(-5)$ & $-199.228(-7)$ & $-199.225(-4)$ & -199.221 \\
\hline $\mathrm{Mg}$ & $-199.616(+438)$ & $-200.048(+6)$ & $-200.052(+2)$ & $-200.050(+4)$ & -200.054 \\
\hline $\mathrm{Ar}$ & $-526.820(+724)$ & $-527.543(+1)$ & $-527.548(-4)$ & $-527.536(+8)$ & -527.544 \\
\hline$\sigma_{\mathrm{rms}}[\mathrm{mH}]$ & 392 & $9.5(3.6)$ & $9.5(3.4)$ & $6.9(4.2)$ & \\
\hline
\end{tabular}


TABLE IV. Ionization energies obtained with Hartree-Fock, second-order perturbation theory for the self-energy (plus the CHF term), FTDA, and the full Faddeev RPA (in hartrees). All results are extrapolated from the cc-p(C)VQZ and cc-p(C)V5Z basis sets (see Table III). The deviations from the experiment (indicated in parentheses) and the rms errors are given in mhartrees. The experimental energies are from Refs. [62-64].

\begin{tabular}{|c|c|c|c|c|c|c|}
\hline & & Hartree-Fock & $\begin{array}{l}\text { Second } \\
\text { order }\end{array}$ & FTDA & FRPA & $\begin{array}{c}\text { Experiment } \\
{[63,64]}\end{array}$ \\
\hline $\mathrm{He}$ & $1 s$ & $0.918(+14)$ & $0.9012(-2.5)$ & $0.9025(-1.2)$ & $0.9008(-2.9)$ & 0.9037 \\
\hline $\mathrm{Be}^{2+}$ & $1 s$ & $5.6672(+116)$ & $5.6542(-1.4)$ & $5.6554(-0.2)$ & $5.6551(-0.5)$ & 5.6556 \\
\hline \multirow[t]{2}{*}{$\mathrm{Be}$} & $2 s$ & $0.3093(-34)$ & $0.3187(-23.9)$ & $0.3237(-18.9)$ & $0.3224(-20.2)$ & 0.3426 \\
\hline & $1 s$ & $4.733(+200)$ & $4.5892(+56)$ & $4.5439(+11)$ & $4.5405(+8)$ & 4.533 \\
\hline \multirow[t]{2}{*}{$\mathrm{Ne}$} & $2 p$ & $0.852(+57)$ & $0.752(-41)$ & $0.8101(+17)$ & $0.8037(+11)$ & 0.793 \\
\hline & $2 s$ & $1.931(+149)$ & $1.750(-39)$ & $1.8057(+24)$ & $1.7967(+15)$ & 1.782 \\
\hline \multirow[t]{2}{*}{$\mathrm{Mg}^{2+}$} & $2 p$ & $3.0068(+56.9)$ & $2.9217(-28.2)$ & $2.9572(+7.3)$ & $2.9537(+3.8)$ & 2.9499 \\
\hline & $2 s$ & 4.4827 & 4.3283 & 4.3632 & 4.3589 & \\
\hline \multirow[t]{2}{*}{$\mathrm{Mg}$} & $3 s$ & $0.253(-28)$ & $0.267(-14)$ & $0.272(-9)$ & $0.280(-1)$ & 0.281 \\
\hline & $2 p$ & $2.282(+162)$ & $2.117(-3)$ & $2.141(+21)$ & $2.137(+17)$ & 2.12 \\
\hline \multirow[t]{3}{*}{$\mathrm{Ar}$} & $3 p$ & $0.591(+12)$ & $0.563(-16)$ & $0.581(+2)$ & $0.579(\approx 0)$ & 0.579 \\
\hline & $3 s$ & $1.277(+202)$ & $1.111(+36)$ & $1.087(+12)$ & $1.065(-10)$ & 1.075 \\
\hline & $3 s$ & & 1.840 & 1.578 & 1.544 & \\
\hline \multicolumn{2}{|c|}{$\sigma_{\mathrm{rms}}[\mathrm{mH}]$} & 81.4 & 29.3 & 13.7 & 10.6 & \\
\hline
\end{tabular}

FRPA explains at least $99 \%$ of the correlation energies, and all calculations, including CCSD, agree with the experiment within the uncertainty expected from basis extrapolation. For $Z \geqslant 10$, the inclusion of RPA correlations predicts about $5 \mathrm{mH}$ more binding than the corresponding FTDA. The atom of Be is the only exception to this trend, as already noted above. In this case the $9 \mathrm{mH}$ difference between FRPA and CCSD is seen also in the basis limit. Based on the agreement between FCI and CCSD in Table I, the remaining discrepancy with the experiment $(\approx 15 \mathrm{mH})$ may be due to the basis set employed, which is probably not capable of accommodating the relevant correlation effects. We have attempted FRPA calculations with the aug-cc-pVXZ bases to allow for a better description of the valence orbits but without any appreciable change in the results.

The Ne atom was also computed in the FRPA approach by using a Hartree-Fock basis with a discretized continuum [36]. The basis set was chosen to be as large as possible to approach the basis-set limit for IEs and EAs but was not optimized for treating core orbits. The total binding energy obtained was $128.888 \mathrm{H}$, away from both the basis-set limit of Table III and the experiment.

Ionization energies are shown in Table IV, together with the predictions from Hartree-Fock theory and the second-order self-energy [obtained by retaining only the first two diagrams of Fig. 1(b)]. Second-order corrections account for a large part of correlations but still lead to sizable errors. The additional correlations included in the present calculations appear to reduce this error substantially. The FTDA [i.e. ADC(3)] results give a measure of the importance of a treatment that is consistent with at least third-order perturbation theory [13]. Corrections are particularly large for states with higher ionization energies, where the density of $2 \mathrm{~h} 1 \mathrm{p}$ states is increased. Since configuration mixing among these states is not introduced by strict second-order perturbation theory, calculations at least at the level of FTDA are required in these cases. Configuration mixing among the $2 \mathrm{~h} 1 \mathrm{p}$ states reduces the errors in the $1 s$ state in Be by a factor of 5. Another effect is the fragmentation of the $3 s$ orbit of Ar. Second-order calculations predict this as a quasiparticle state $36 \mathrm{mH}$ away from the empirical energy and carrying 0.81 of the total orbit's intensity. A small satellite state with relative intensity of 0.10 is calculated at larger separation energies. The mixing with $2 \mathrm{~h} 1 \mathrm{p}$ configurations corrects the energies of both peaks and redistributes their strengths more correctly. For the FRPA calculation the peak at $1.065 \mathrm{H}$ has intensity of 0.61 , close to the experimental values (peak at $1.075 \mathrm{H}$ with intensity 0.55 [62]). The second peak is obtained at $1.544 \mathrm{H}$ and carries the remaining strength of the original quasiparticle.

Adding the effects of RPA excitations has a larger impact on ionization than on correlation energies. Almost all the calculated IEs shift closer to the experimental values by a few millihartree. The only exceptions are the two-electron He atom, where the RPA approach tends to overestimate correlations, and the first ionization of Be, where soft excitations tend to invalidate the RPA. In general, the rms error for the valence orbits of Table IV decreases from 13.7 to $10.6 \mathrm{mH}$, passing from FTDA to FRPA.

The FRPA first and second IEs of the Ne atom computed using the discretized continuum basis of Ref. [36] are 0.801 and $1.795 \mathrm{H}$. These are in good agreement with the extrapolations of Table IV and give us further confidence in applying Eq. (7) also for quasiparticle states.

\section{CONCLUSIONS AND DISCUSSION}

We have performed microscopic calculations of total and ionization energies in order to assess the accuracy of the Faddeev RPA approach for light atoms. The FRPA is an expansion of the many-body self-energy that makes explicit the coupling between particles and collective excitations arising from interacting electrons and holes. This formalism completely includes the $\mathrm{ADC}(3)$ theory and retains all contributions from perturbation theory up to third order, which is crucial for a 
correct prediction of IEs for outer-valence electrons in atomic and molecular systems. At the same time, it also includes full resummations of RPA diagrams necessary to screen the long-range Coulomb interaction in extended systems [5,6]. In this work the FRPA was tested in the basis-set limit, and it was found that even for very small systems, where RPA violations of the Pauli principle might have hindered satisfactory results, the FRPA is accurate.

In general, FTDA [i.e. ADC(3)] and FRPA give very similar results for the lightest systems, while the inclusion of ground-state correlations via RPA theory leads to small but systematic improvements as the atomic number increases. For total binding energies, their difference is negligible in the $\mathrm{He}$ and $\mathrm{Be}$ atoms, while the FRPA yields $\approx 5 \mathrm{mH}$ more correlation energy for atomic numbers $Z \geqslant 10$. Except for the lightest atoms, $99 \%$ of the the total correlation energy is normally recovered, and the total energies obtained agree well with CCSD (as expected from the estimates for ADC(3) in Ref. [20]). The discrepancies with the experimental data are also within the errors estimated for the extrapolation to the basis-set limit. The only notable exception is the neutral Be atom, for which the small gap at the Fermi surface complicates the extraction of the correlation energy. In this case, the discrepancy obtained with respect to the experiment appears to be mostly due to deficiencies in the basis set. However, a smaller fraction of it is probably related to missing correlations.

Similar trends are found for the ionization energies. For the two-electron cases, $\mathrm{He}$ and $\mathrm{Be}^{2+}$, FRPA does not introduce improvements with respect to FTDA, but it gives again sensible predictions. The above problems in describing the correlations in neutral $\mathrm{Be}$ are also reflected in the results for the first ionization energy. For all other cases, the use of RPA shifts IEs by $2-10 \mathrm{mH}$ and always brings them closer to the experiment. On average, the rms error for outer-valence IEs decreases from 13.7 to $10.6 \mathrm{mH}$ by adding RPA effects. The $3 s$ orbit in $\mathrm{Ar}$ is found to be fragmented, and configuration mixing effects between $2 \mathrm{~h} 1 \mathrm{p}$ states are required to obtain the correct ionization energy and relative intensity. The present results are in agreement with analogous calculations of simple molecules [44] and show that conclusions on the accuracy of the FRPA method still hold in the basis-set limit.

Numerically, the FRPA can be implemented as a diagonalization in 2p1h-2h1p space, implying similar computational costs as an $\operatorname{ADC}(3)$ calculation. Due to the inclusion of RPA excitations, the FRPA method holds the promise of bridging the gap between accurate descriptions of quasiparticles in finite and extended systems. Investigating the feasibility of FRPA for larger molecules and the electron gas is therefore a priority for future research efforts. Consistent calculations of quasiparticle properties in these cases, once feasible, could also be useful for constraining functionals in quasiparticle density functional theory [65].

\section{ACKNOWLEDGMENTS}

This work was supported by the Japanese Ministry of Education, Science and Technology (MEXT) under KAKENHI Grant No. 21740213 and by the United Kingdom Science and Technology Facilities Council (STFC) under Grant No. ST/J000051. M.D. acknowledges support from FWO-Flanders. D.V.N. and M.D. acknowledge support from the QCMM Alliance Ghent-Brussels.
[1] T. Helgaker et al., J. Phys. Org. Chem. 17, 913 (2004).

[2] R. J. Bartlett and M. Musiał, Rev. Mod. Phys. 79, 291 (2007).

[3] J. Linderberg and Y. Öhrn, Propagators in Quantum Chemistry (Wiley, Hoboken, NJ, 2004).

[4] A. L. Fetter and J. D. Walecka, Quantum Theory of ManyParticle Physics (McGraw-Hill, New York, 1971).

[5] R. D. Mattuck, A Guide to Feynman Diagrams in the Many-Body Problem (McGraw-Hill, New York, 1967).

[6] W. H. Dickhoff and D. Van Neck, Many-Body Theory Exposed! (World Scientific, Singapore, 2005).

[7] L. S. Cederbaum and W. Domcke, Adv. Chem. Phys. 36, 205 (1977).

[8] W. von Niessen, J. Schirmer, and L. S. Cederbaum, Comput. Phys. Rep. 1, 57 (1984).

[9] V. G. Zakrzewski and W. von Niessen, J. Comput. Chem. 14, 13 (1993).

[10] J. V. Ortiz, Computational Chemistry: Reviews of Current Trends, edited by J. Leszczyinski (World Scientific, Singapore, 1997), Vol. 2, p. 1.

[11] D. Danovich, Encyclopedia of Computational Chemistry, (Wiley, New York, 1998), Vol. 2, pp. 1190-1202.

[12] V. G. Zakrzewski, O. Dolgounitcheva, and J. V. Ortiz, J. Chem. Phys. 129, 104306 (2008).

[13] O. Walter and J. Schirmer, J. Phys. B 14, 3805 (1981).
[14] L. S. Cederbaum, J. Schirmer, W. Domcke, and W. von Niessen, Adv. Chem. Phys. 65, 115 (1986).

[15] S. Knippenberg, J.-P. François, and M. S. Deleuze, J. Comp. Chem. 27, 1703 (2006).

[16] J. Schirmer, Phys. Rev. A 26, 2395 (1982).

[17] J. Schirmer, L. S. Cederbaum, and O. Walter, Phys. Rev. A 28, 1237 (1983).

[18] J. Schirmer, Phys. Rev. A 43, 4647 (1991).

[19] F. Mertins and J. Schirmer, Phys. Rev. A 53, 2140 (1996).

[20] A. B. Trofimov and J. Schirmer, J. Chem. Phys. 123, 144115 (2005).

[21] M. Pernpointner, J. Chem. Phys. 121, 8782 (2004).

[22] A. B. Trofimov, G. Stelter, and J. Schirmer, J. Chem. Phys. 117, 6402 (2002).

[23] J. H. Starcke, M. Wormit, and A. Drew, J. Chem. Phys. 130, 024104 (2009).

[24] L. Hedin, Phys. Rev. 139, A796 (1965).

[25] F. Aryasetiawan and O. Gunnarsson, Rep. Prog. Phys. 61, 237 (1998).

[26] G. Onida, L. Reining, and A. Rubio, Rev. Mod. Phys. 74, 601 (2002).

[27] U. von Barth and B. Holm, Phys. Rev. B 54, 8411 (1996).

[28] B. Holm, Phys. Rev. Lett. 83, 788 (1999).

[29] E. L. Shirley, Phys. Rev. B 54, 7758 (1996). 
[30] Y. Dewulf, D. Van Neck, and M. Waroquier, Phys. Rev. B 71, 245122 (2005).

[31] M. Springer, F. Aryasetiawan, and K. Karlson, Phys. Rev. Lett. 80, 2389 (1998).

[32] P. Romaniello, S. Guyot, and L. Reining, J. Chem. Phys. 131, 154111 (2009).

[33] J. F. Dobson, in Topics in Condensed Matter Physics, edited by M. P. Das (Nova, New York, 1994).

[34] M. Dion, H. Rydberg, E. Schröder, D. C. Langreth, and B. I. Lundqvist, Phys. Rev. Lett. 92, 246401 (2004).

[35] G. Román-Pérez and J. M. Soler, Phys. Rev. Lett. 103, 096102 (2009).

[36] C. Barbieri, D. Van Neck, and W. H. Dickhoff, Phys. Rev. A 76, 052503 (2007).

[37] W. H. Dickhoff and C. Barbieri, Prog. Part. Nucl. Phys. 52, 377 (2004).

[38] C. Barbieri, C. Giusti, F. D. Pacati, and W. H. Dickhoff, Phys. Rev. C 70, 014606 (2004).

[39] C. Barbieri, Phys. Lett. B 643, 268 (2006).

[40] C. Barbieri and M. Hjorth-Jensen, Phys. Rev. C 79, 064313 (2009).

[41] C. Barbieri, Phys. Rev. Lett. 103, 202502 (2009).

[42] P. Danielewicz and P. Schuck, Nucl. Phys. A 567, 78 (1994).

[43] Y. Dewulf, D. Van Neck, and M. Waroquier, Phys. Rev. C 65, 054316 (2002).

[44] M. Degroote, D. Van Neck, and C. Barbieri, Phys. Rev. A 83, 042517 (2011).

[45] C. Froese-Fischer, J. Phys. B 26, 855 (1993).

[46] C. Froese-Fischer and P. Jonsson, Comput. Phys. Commun. 84, 37 (1994).
[47] C. Froese-Fischer, T. Brage, and P. Johnsson, Computational Atomic Structure: An MCHF Approach (Taylor and Francis, London, 1997).

[48] D. Sundholm and J. Olsen, Phys. Rev. A 47, 2672 (1993).

[49] D. Sundholm and J. Olsen, J. Chem. Phys. 98, 7152 (1993).

[50] D. Sundholm and J. Olsen, Phys. Rev. A 49, 3453 (1994).

[51] K. L. Sharkey, S. Bubin, and L. Adamowicz, J. Chem. Phys. 132, 184106 (2010).

[52] C. Hättig, Adv. Quantum Chem. 50, 37 (2005).

[53] G. E. Scuseria, T. M. Henderson, and D. C. Sorensen, J. Chem. Phys. 129, 231101 (2008).

[54] S. Ethofer and P. Schuck, Z. Phys. 228, 264 (1969).

[55] J. Winter, Nucl. Phys. A 194, 535 (1972).

[56] C. Barbieri and W. H. Dickhoff, Phys. Rev. C 63, 034313 (2001).

[57] E. R. Davidson, S. A. Hagstrom, S. J. Chakravorty, V. M. Umar, and C. F. Fischer, Phys. Rev. A A44, 7071 (1991).

[58] S. J. Chakravorty and E. R. Davidson, J. Phys. Chem. 100, 6167 (1996).

[59] G. Martin [http://www.weizmann.ac.il/oc/martin/atoms.shtml].

[60] M. Pernpointner, J. Chem. Phys. 121, 8782 (2004).

[61] C. Barbieri and W. H. Dickhoff, Phys. Rev. C 68, 014311 (2003).

[62] I. E. McCarthy, R. Pascual, P. Storer, and E. Weigold, Phys. Rev. A 40, 3041 (1989).

[63] NIST Atomic Spectra Database, NIST Standard Reference Database 78 [http://physics.nist.gov/PhysRefData/ASD/ index.html].

[64] A. Thompson et al., X-ray Data Booklet (Lawrence Berkeley National Laboratory, Berkeley, CA, 2001), and references therein.

[65] D. Van Neck, S. Verdonck, G. Bonny, P. W. Ayers, and M. Waroquier, Phys. Rev. A 74, 042501 (2006). 